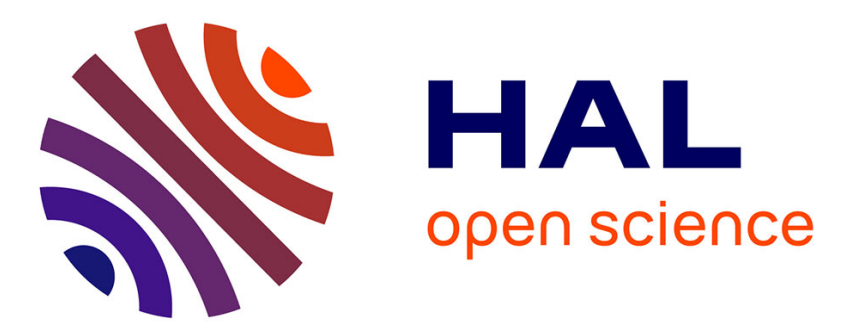

\title{
A Numerical Method for Solving Fractional-Order Viscoelastic Euler-Bernoulli Beams
}

Chunxiao Yu, Jie Zhang, Yiming Chen, Yujing Feng, Aimin Yang

\section{To cite this version:}

Chunxiao Yu, Jie Zhang, Yiming Chen, Yujing Feng, Aimin Yang. A Numerical Method for Solving Fractional-Order Viscoelastic Euler-Bernoulli Beams. Chaos, Solitons \& Fractals, 2019, 128, pp.275279. 10.1016/j.chaos.2019.07.035 . hal-02884958

\section{HAL Id: hal-02884958 https://hal.science/hal-02884958}

Submitted on 30 Jun 2020

HAL is a multi-disciplinary open access archive for the deposit and dissemination of scientific research documents, whether they are published or not. The documents may come from teaching and research institutions in France or abroad, or from public or private research centers.
L'archive ouverte pluridisciplinaire HAL, est destinée au dépôt et à la diffusion de documents scientifiques de niveau recherche, publiés ou non, émanant des établissements d'enseignement et de recherche français ou étrangers, des laboratoires publics ou privés. 


\title{
A Numerical Method for Solving Fractional-Order Viscoelastic Euler-Bernoulli Beams
}

\author{
Chunxiao $\mathrm{Yu}^{1 *}$, Jie Zhang ${ }^{1}$, Yiming Chen ${ }^{1}$, Yujing Feng ${ }^{1}$, Aimin Yang ${ }^{2 *}$ \\ ${ }^{1}$ School of Science, Yanshan University, Qinhuangdao 066004, China \\ ${ }^{2}$ College of Science, North China University of Science and Technology, Tangshan 063000, China \\ *Email of Corresponding Author: chxy@ysu.edu.cn (Chunxiao Yu); yangaimin_may@163.com
}

(Aimin Yang)

\begin{abstract}
In this paper, a new method is presented for solving the constitutive equations of fractional-order viscoelastic Euler-Bernoulli beams. Firstly, the constitutive equation of the Euler-Bernoulli beam is established by analyzing the constitutive relation between the fractional viscoelastic materials. Secondly, the constitutive equation of the beam is transformed into a matrix equation by using a Quasi-Legendre polynomial in the time domain. Then the matrix equation is discretized and solved, and the numerical solution is obtained for the constitutive equation of the beam. Finally, numerical analysis of two different fractional viscoelastic materials is carried out by numerical experiments. Displacements under different external loads are obtained for the polybutadiene beam and butyl B252 beam. With the change of time and position, the change law of displacements is found. And the performance of the two materials is compared and analyzed.
\end{abstract}

Keyword: Viscoelastic Euler-Bernoulli simply supported beam; fractional derivative constitutive relation; Quasi-Legendre polynomial; numerical solution; operator matrix

\section{Introduction}

Since viscoelastic materials have a good damping effect, they have been widely used in engineering calculations. In the classical elastic mechanics analysis, different models can be obtained by series or parallel connection of springs and sticks [1], and the time domain problem can be converted to a frequency domain problem using Fourier transform or Laplace transform. The relaxation function and the creep function are obtained experimentally, and the viscoelastic properties are analyzed by frequency and amplitude [2]. With the development of fractional order methods, scholars begin to use fractional order to describe the constitutive equation of viscoelasticity. For example, the fractional derivative model [3-5], each model can also be converted to each other [6-8]. Since fractional order equations have good memory and can be used to describe material properties more accurately with fewer parameters, they are considered to be good mathematical models for describing the dynamic mechanical behavior of materials.

In the 1930s, Gement first used the fractional derivative to describe the constitutive relation of viscoelastic materials [9], but it was not until the 1980s that the constitutive relations of fractional derivatives were widely valued $[10,11]$. Huanying proposed the fractional-order constitutive relation model represented by the fractional element networ [12], and the corresponding constraint problem of nonlinear optimization was solved by the interior point algorithm. In Ref. [13], the dynamic analysis of nonlinear viscoelastic plates subjected to external loads was carried out. A multiscale approach was used to solve the equation of motion to determine the primary and secondary harmonic resonance 
response curves.

For the dynamic analysis of viscoelastic materials, many scholars have proposed related numerical methods. The common methods of viscoelastic structure analysis in the literature are the differential method and the finite element method [14-18]. There are also the Galerkin method [19], the variational iteration method [20], the multiscale method and so on [21, 22].

For the governing equations of the fractional viscoelastic Euler-Bernouli beam, the majority of existing literatures evaluate the solvability, but they all fail to give the specific numerical solutions. On this basis, a method of Quasi-Legendre polynomial will be used to solve the governing equations [23, 24]. Because the displacement of the beam subjected to external loads is not only related to the position of the beam, but also is related to time, the influence of time on the displacements is considered for the first time in this paper. And the dynamic behavior of the beam is more fully described and analyzed, which has important practical significance.

\section{Establishment of constitutive equation for Euler-Bernoulli beam}

In order to better understand the mechanical properties of viscoelastic materials, some theoretical knowledge of viscoelasticity is given firstly, and the constitutive equations of Euler-Bernoulli beams are derived.

The general linear integer order differential equation of viscoelasticity is described by the following relationship.

$$
\sum_{k=0}^{n} a_{k} \frac{\mathrm{d}^{k} \sigma(t)}{\mathrm{d} t^{k}}=\sum_{k=0}^{n} b_{k} \frac{\mathrm{d}^{k} \varepsilon(t)}{\mathrm{d} t^{k}}, m, n=0,1,2, \cdots
$$

The differential equations shown in equation (1) are complex and difficult to solve. A more efficient way to maintain the linearity of the model is the application of fractional viscoelastic models. A fractional model can be expressed as

$$
\sum_{k=0}^{n} a_{k} D^{\alpha_{k}} \sigma(t)=\sum_{k=0}^{m} b_{k} D^{\beta_{k}} \varepsilon(t)
$$

The fractional model is defined as follows.

$$
\sigma=\left(E_{\varepsilon}+E_{t_{\alpha}} \frac{\mathrm{d}^{\alpha} \varepsilon(t)}{\mathrm{d} t}\right)=E\left(\varepsilon+\mu_{\alpha} \frac{\mathrm{d}^{\alpha} \varepsilon(t)}{\mathrm{d} t}\right)
$$

where $\mu_{\alpha}=E_{t_{\alpha}} / E, E$ is the Young's modulus of the beam material, and $E_{t_{\alpha}}$ is the damping coefficient.

The fractional derivative of order $\alpha$ is defined as below.

$$
\frac{\mathrm{d}^{\alpha}}{\mathrm{d} t} f(t) \equiv{ }_{a} D_{t}^{\alpha} f(t) \equiv \frac{1}{\Gamma(1-\alpha)} \frac{\mathrm{d}}{\mathrm{d} t} \int_{a}^{t} \frac{f(\tau)}{(t-\tau)^{\alpha+1+m}} \mathrm{~d} \tau
$$

For many real materials, the range of the fractional derivative order $\alpha$ is $0<\alpha \leq 1$, while the lower limit of the integral is $a=0$.

Under the assumption of Bernoulli-Euler theory, the moment of inertia and shear deformation are neglected, the bending stiffness and mass density are assumed to be uniform [25], and the governing equation of the forced lateral motion of the beam is obtained.

$$
E J\left(\frac{\partial^{4} f(x, t)}{\partial x^{4}}+\mu_{\alpha} \frac{d^{\alpha}}{d t}\left(\frac{\partial^{4} f(x, t)}{\partial x^{4}}\right)\right)+A \rho \frac{\partial^{2} f(x, t)}{\partial t^{2}}=p(x, t)
$$

where $\mathrm{A}$ is the cross-sectional area of the beam, $\mathrm{J}$ is the moment of inertia of the beam section, $\rho$ is the material mass density of the material, $f(x, t)$ is the lateral displacement of the neutral beam axis, $t$ is 
time, $x$ is the longitudinal coordinate, and $p(x, t)$ is the external load.

\section{Solution of constitutive equation with Quasi-Legendre polynomial}

In this section, some properties of the Quasi-Legendre polynomial are given. Using these properties, the constitutive equations are solved by function approximations and operator matrix analysis.

\subsection{Definition and properties of the Quasi-Legendre polynomial}

When the polynomial is used to approximate the unknown function and solve the fractional differential equation, the historical inheritance and dependence of the fractional differential and integral are not well reflected. To overcome this deficiency, we improve the Legendre polynomial $L_{n}(t)$ and construct the following Quasi- Legendre polynomial.

Definition 1: In the shifted Legendre polynomial

$$
L_{n}(t)=(n !) \frac{\mathrm{d}^{n}}{\mathrm{~d} t^{n}}\left[\left(t^{2}-t\right)^{n}\right], t \in[0,1], n=1,2, \cdots
$$

let $t=z^{\alpha}, \alpha>0$, and we have $L_{n}(t)=L_{n}\left(z^{\alpha}\right)$, which can be abbreviated as $L_{n}\left(z^{\alpha}\right)=F l_{n}^{\alpha}(z) . F l_{n}^{\alpha}(z)$

is called a Quasi-Legendre polynomial.

For convenience, the argument $z$ is replaced by $x$, and the lower indicator $i$ is replaced by $n$. Then the Quasi-Legendre polynomial $F l_{n}^{\alpha}(z)$ is expressed as $F l_{i}^{\alpha}(x)$.

The recursive relationship of $F l_{i}^{\alpha}(x)$ is

$$
\begin{gathered}
F l_{i+1}^{\alpha}(x)=\frac{(2 i+1)\left(2 x^{\alpha}-1\right)}{i+1} F l_{i}^{\alpha}(x)-\frac{i}{i+1} F l_{i-1}^{\alpha}(x) \\
F l_{0}^{\alpha}(x)=1, F l_{1}^{\alpha}(x)=2 x^{\alpha}-1
\end{gathered}
$$

The analytical form of $F l_{i}^{\alpha}(x)$ is

$$
F l_{i}^{\alpha}(x)=\sum_{s=0}^{i} b_{s, i} x^{s \alpha}
$$

where $b_{s, j}=\frac{(-1)^{i+s}(i+s) !}{(i+s) !(s !)^{2}}, F l_{i}^{\alpha}(0)=(-1)^{i}, F l_{i}^{\alpha}(1)=1$.

\subsection{Function approximation}

For the function $f(x, t) \in L^{2}([0,1] \times[0,1])$, it can be expanded to

$$
f(x, t)=\sum_{i=0}^{\infty} \sum_{j=0}^{\infty} u_{i j} F l_{i}^{\alpha}(x) F l_{j}^{\beta}(t)
$$

where

$$
u_{i j}=(2 i+1)(2 j+1) \alpha \beta \times \int_{0}^{1} \int_{0}^{1} u(x, t) F l_{i}^{\alpha}(x) F l_{i}^{\beta}(t) \omega_{l}^{\beta}(x) \omega_{l}^{\beta}(t) \mathrm{d} x \mathrm{~d} t, i, j=0,1,2, \cdots
$$

In Equation (3), finite terms are often considered to find numerical solutions. Equation (3) can be rewritten as

$$
f(x, t) \approx \sum_{i=0}^{m-1} \sum_{j=0}^{n-1} u_{i j} F l_{i}^{\alpha}(x) F l_{j}^{\beta}(t)
$$

Equation (4) can be expressed as the following matrix.

$$
f(x, t) \approx \sum_{i=0}^{m-1} \sum_{j=0}^{n-1} u_{i j} F l_{i}^{\alpha}(x) F l_{i}^{\beta}(t)=\Phi(x) U \Phi(t)
$$

where 


$$
\begin{gathered}
U=\left\{u_{i j}\right\}_{i, j=0}^{m-1 . n-1} \\
\Phi(x)=\left[F l_{0}^{\alpha}(x), F l_{1}^{\alpha}(x), \cdots F l_{m-1}^{\alpha}(x)\right] \\
\Phi(t)=\left[F l_{0}^{\beta}(t), F l_{1}^{\beta}(t), \cdots, F l_{n-1}^{\beta}(t)\right]
\end{gathered}
$$

\subsection{Operator matrix}

The differential form of the vector function $\Phi(x)$ can be approximated as a matrix-vector product.

$$
D^{\gamma} \Phi(x)=D^{\gamma} \Phi(x)
$$

The elements in $D^{\gamma}$ are

$$
\left\{d_{i j}\right\}_{i, j}^{m-1, m-1}=(2 j+1) \alpha \sum_{s=0}^{i} \sum_{r=0}^{j} b_{r, j} b_{s, i}^{\prime} \frac{\Gamma(s \alpha+1)}{\Gamma(s \alpha-\gamma+1)} \frac{1}{(s+r+1) \alpha-\gamma}
$$

Where

$$
b_{s, j}^{\prime}=\left\{\begin{array}{l}
0, s \alpha \in N_{0} \text { and } s \alpha<\gamma \\
b_{s, j}, s \alpha \notin N_{0} \text { and } s \alpha \geq[\gamma] \text { or } s \alpha \in N_{0} \text { and } s \alpha \geq \gamma
\end{array}\right.
$$

Using Equations (4) and (5), Equation (2) is solved. Then we have

$$
\begin{gathered}
\frac{\partial^{4} f(x, t)}{\partial x^{4}} \approx \frac{\partial^{4}\left(\Phi^{T}(x) U \Phi(t)\right)}{\partial x^{4}}=\left(\frac{\partial^{4} \Phi(x)}{\partial x^{4}}\right)^{T} U \Phi(x) \approx \Phi^{T}(x)\left(D^{4}\right)^{T} U \Phi(t) \\
\frac{\partial^{2} f(x, t)}{\partial t^{2}} \approx \frac{\partial^{2}\left(\Phi^{T}(x) U \Phi(t)\right)}{\partial t^{2}} \approx \Phi^{T}(x) U D^{2} \Phi(t) \\
\frac{d^{\alpha}}{d t}\left(\frac{\partial^{4} f(x, t)}{\partial x^{4}}\right)=D_{t}^{\alpha} \frac{\partial^{4} f(x, t)}{\partial x^{4}} \approx D_{t}^{\alpha} \frac{\partial^{4}\left(\Phi^{T}(x) U \Phi(x)\right)}{\partial x^{4}}=D_{t}^{\alpha} \Phi^{T}(x)\left(D^{4}\right)^{T} U \Phi(t) \\
=\Phi^{T}(x)\left(D^{4}\right)^{T} U D_{t}^{\alpha} \Phi(t) \approx \Phi^{T}(x)\left(D^{4}\right)^{\alpha} \Phi(t)
\end{gathered}
$$

Equation (2) can be transformed into an algebraic equation as follows.

$$
E J \Phi(x)\left[D^{4}\right]^{T} \Phi(t)+\mu_{\alpha} \Phi(x)\left[D^{4}\right]^{T} U D^{\alpha} \Phi(t)+A \rho \Phi(x) U D^{2} \Phi(t)-p(x, t)=0(7)
$$

Using the configuration method, $(x, t)$ is discretized to $\left(x_{i}, t_{i}\right)$, so that Equation (7) becomes a linear system of equations. Then the numerical solution of the original problem can be found.

\section{Numerical examples}

In this section, the newly proposed method is used to simulate two different materials. And some important information is obtained by numerical analysis.

\subsection{Simulation of polybutadiene beam}

The length of the beam is taken as $l=1 \mathrm{~m}$, the density $\rho_{l}=160 \mathrm{~kg} / \mathrm{m}^{3}$, the moment of inertia $J=(0.1)^{4} / 12$, and the cross-sectional area $A=0.72 \mathrm{~m}^{2}$. The related material parameters are taken as $\alpha=0.528, E=8.14 \times 10^{5}, \quad \mu_{\alpha}=7.31 / 81.4$, respectively. For Equation (2), suppose that both ends of the beam are fixed, and the given boundary condition is expressed as 


$$
f(x, t)=0, \frac{\partial f(x, 0)}{\partial x}=0, x=0, l
$$

The initial condition is as follows.

$$
f(x, 0)=0, \frac{\partial f(x, 0)}{\partial t}=0
$$

For Equation (4), take $m=6, n=6$. When the external load $p(x, t)$ is taken as different values, the numerical solution is shown in Figure 1.

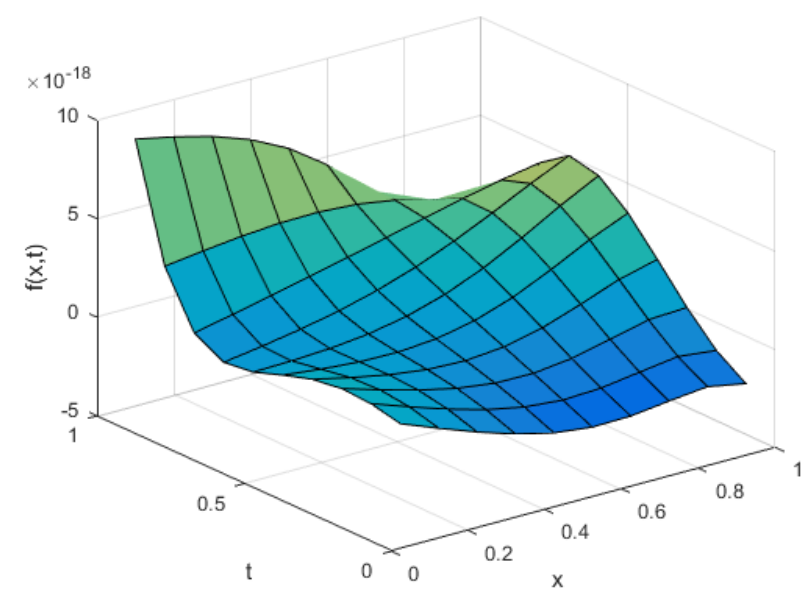

(a) Displacements $(p(x, t)=0)$

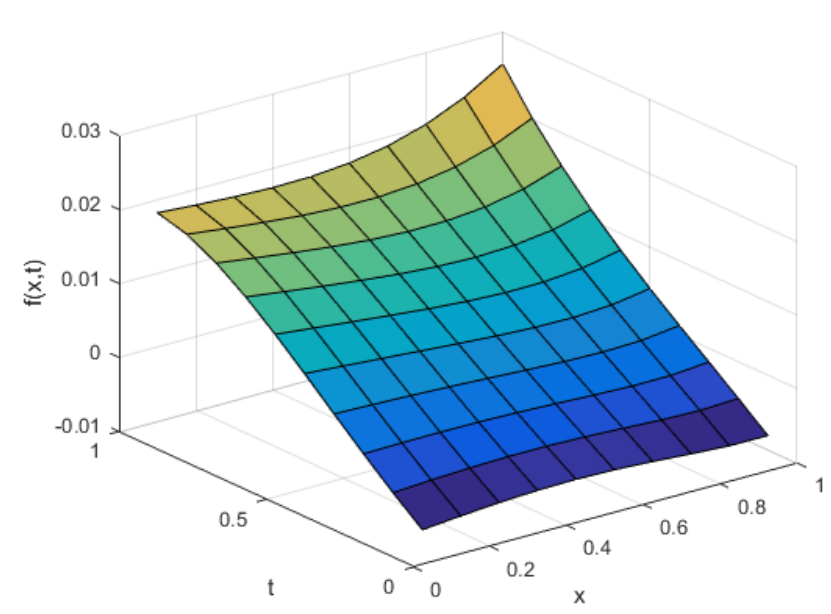

(b) Displacements $(p(x, t)=20$ Heaviside $(t))$ 


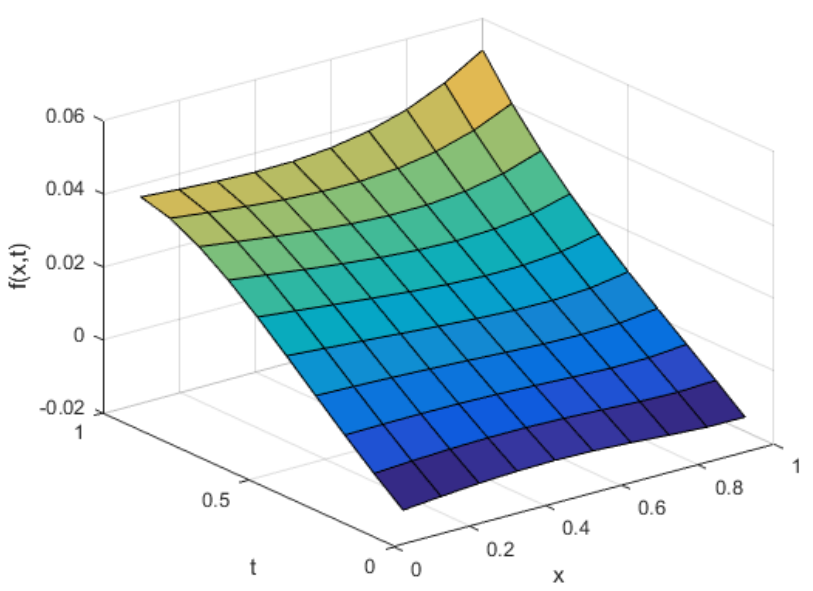

(c) Displacements $(p(x, t)=40$ Heaviside $(t))$

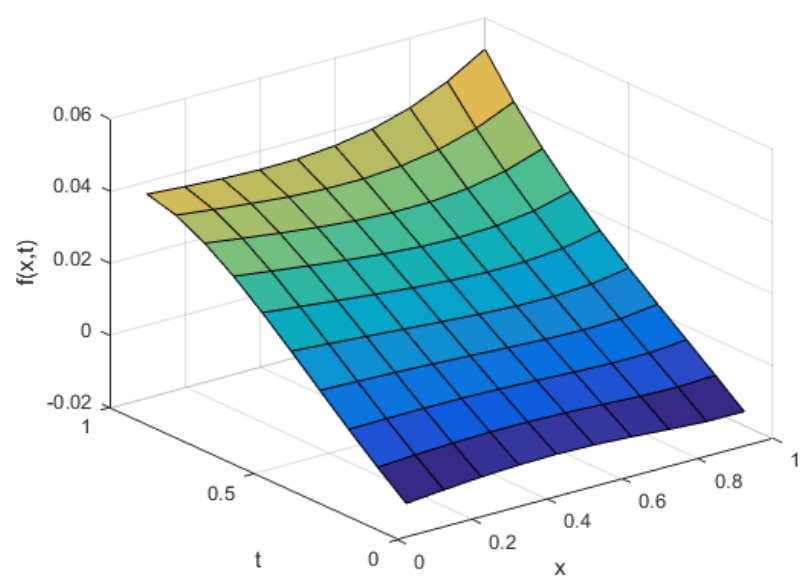

(d) Displacements $(p(x, t)=\sin (\pi x) \sin (t))$

Figure 1 Numerical Solution under different external loads

\subsection{Simulation of butyl B252 beam}

The length of the beam is taken as $1=1 \mathrm{~m}$, the moment of inertia $J=(0.1)^{4} / 12$, the cross-sectional area $A=0.72 \mathrm{~m}^{2}$. The parameters are taken as $\alpha=0.519, E=1.05 \times 10^{6}, E_{t_{\alpha}}=2.44 \times 10^{5}$, $\rho_{l}=200 \mathrm{~kg} / \mathrm{m}^{3}$, respectively. The boundary condition, initial condition and values of $\mathrm{m}$ and $\mathrm{n}$ are the same as those in Section 4.1.

When the external load is taken as different values, the numerical solution of Equation (7) is shown in Figure 2. 


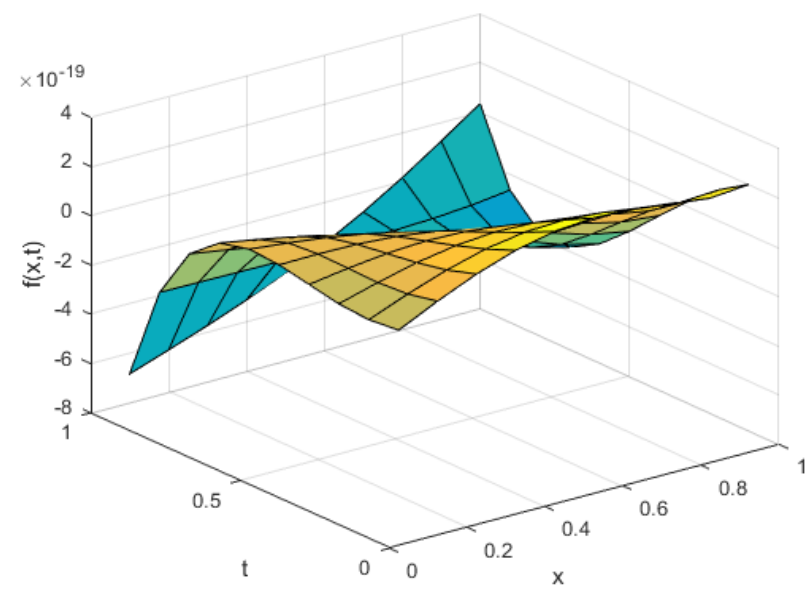

(a) Displacements $(p(x, t)=0)$

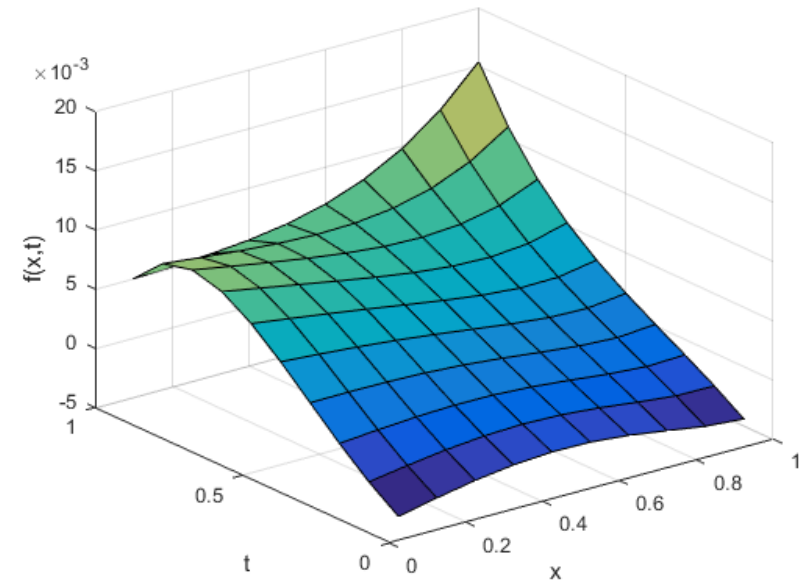

(b) Displacements $(p(x, t)=20$ Heaviside $(t))$

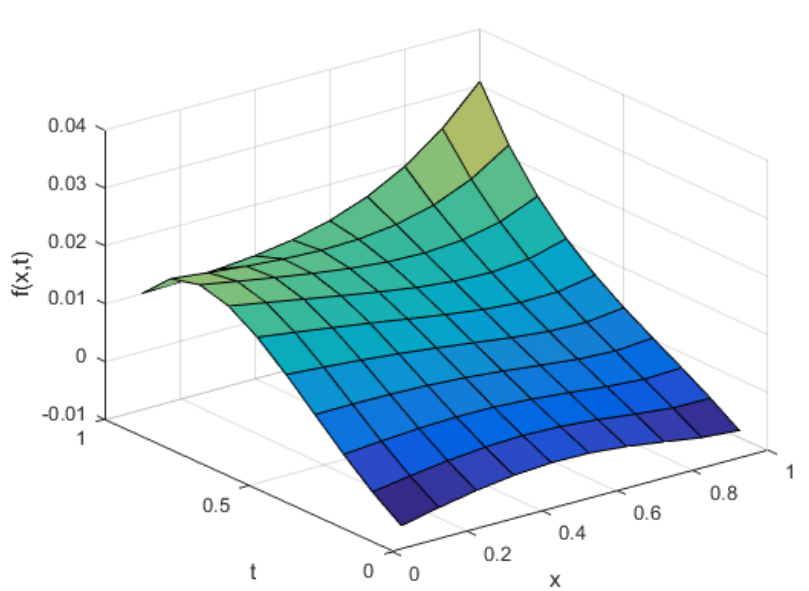

(c) Displacements $(p(x, t)=40$ Heaviside $(t))$ 


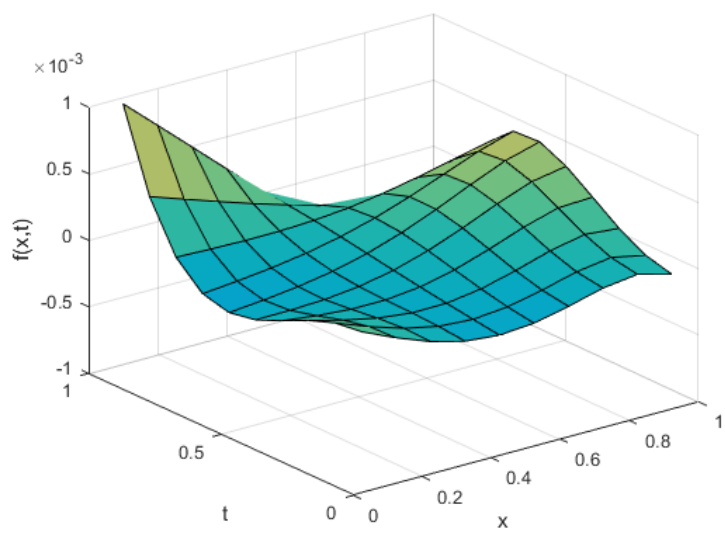

(d) Displacements $(p(x, t)=\sin (\pi x) \sin (t))$

\section{Figure 2 Numerical Solution under different external loads}

It is known from Figure 2 that as time $t$ and position $x$ change, the displacement becomes larger and larger, and it gradually becomes stable.

Numerical experiments show that the displacements of the beam are larger and more stable with the change of time and position. The larger the damping amplitude of the viscoelastic beam, the better the performance of the material. Under the same load, the displacements of butyl B252 beam are smaller than those of the polybutadiene beam, and the damping effect is much better.

\section{Conclusion}

According to the constitutive relation of fractional-order viscoelastic beams, the constitutive equations of Euler-Bernoulli beams are established. The constitutive equations are transformed into matrix equations by the Quasi-Legendre polynomial. The numerical solutions are obtained for the constitutive equations of Euler-Bernoulli beams under different loads. And performances of two fractional viscoelastic materials are analyzed by numerical experiments. Using the Quasi-Legendre polynomial, the solution of the constitutive equation of the beam can be implemented directly in the time domain, which greatly simplifies the solution process.

\section{Acknowledgements}

The work in this research is supported by the National Natural Science Foundation of China (No. 11301459) and Natural Science Foundation of Hebei Province in China (No. A2017203100). The authors also gratefully acknowledge the support of the LE STUDIUM RESEARCH PROFESSORSHIP award of Centre-Val de Loire region in France.

\section{References}

[1] I. Podlubny. Fractional-order systems and $\mathrm{PI} / \mathrm{sup} / \mathrm{spl}$ lambda//D/sup /spl mu//-controllers. Automatic Control IEEE Transactions on, 1999, 44(1): 208-214.

[2] S.N. Mahmoodi, N. Jalili, S.E. Khadem. An experimental investigation of nonlinear vibration and frequency response analysis of cantilever viscoelastic beams. Journal of Sound \& Vibration, 2008, 311(3): 1409-1419.

[3] A.Y.T. Leung, H.X. Yang, P. Zhu, Z.J. Guo. Steady state response of fractionally damped nonlinear viscoelastic arches by residue harmonic homotopy. Computers \& Structures, 2013, 121(5): 10-21.

[4] A.C. Galucio, J.F. Deü, R. Ohayon. Finite element formulation of viscoelastic sandwich beams using fractional derivative operators. Computational Mechanics, 2004, 33(4): 282-291. 
[5] R. Lewandowski, A. Bartkowiak, H. Maciejewski. Dynamic analysis of frames with viscoelastic dampers: a comparison of damper models. Structural Engineering \& Mechanics, 2012, 41(1): 113-137.

[6] P.K. Pandey, S.S.A. Jaboob. A finite difference method for a numerical solution of elliptic boundary value problems. Applied Mathematics \& Nonlinear Sciences, 2018, 3(1): 311-320.

[7] W. Gao, W. Wang. A tight neighborhood union condition on fractional (g, f,n ',m)-critical deleted graphs. Colloquium Mathematicum, 2017, 149(2): 291-298.

[8] R. Xiao, H.G. Sun, W. Chen. An equivalence between generalized Maxwell model and fractional Zener model. Mechanics of Materials, 2016, 100: 148-153.

[9] A. Gemant. On fractional differences. Phil Mag, 1938, 25(168): 540-549.

[10] R.L. Bagley, P.J. Torvik. On the fractional calculus model of viscoelasticity behavior. Journal of Rheology, 1986, 30(1): 133-155.

[11] R.C. Koeller. Applications of the fractional calculus to the theory of viscoelasticity. Transactions of the Asme Journal Applied, 1984, 51(3): 294-298.

[12] H.Y. Xu, X.J. Jiang. Creep constitutive models for viscoelastic materials based on fractional derivatives. Computers and Mathematics with Applications, 2017, 73(6): 1377-1384.

[13] Y. Davood, N. Hamed. Frequency analysis of the nonlinear viscoelastic plates subjected to subsonic flow and external loads. Thin-Walled Structures, 2015, 92: 65-75.

[14] C. Chazal, R.M. Pitti. Integral approach for time dependent materials using finite element method. Journal of Theoretical \& Applied Mechanics, 2017, 4(4): 1029-1048.

[15] J.N. Reddy. An introduction to continuum mechanics: With applications. Cambridge University Press, 2008.

[16] R.J. Theodore, J.H. Arakeri, A. Ghosal. The modeling of axially translating flexible beams. Journal of Sound \& Vibration, 1996, 191(3): 363-376.

[17] S.B.G. Karakoc, Y. Ucar, N. Yagmurlu. Numerical solutions of the mrlw equation by cubic b-spline galerkin finite element method. Kuwait Journal of Science, 2015, 42(2): 141-159.

[18] N.H. Zhang, C.J. Cheng. Non-linear mathematical model of viscoelastic thin plates with its applications. Computer Methods in Applied Mechanics \& Engineering, 1998, 165(1-4): 307-319.

[19] Y. Lei, M.I. Friswell, S. Adhikari. A Galerkin method for distributed systems with non-local damping. International Journal of Solids \& Structures, 2006, 43(11): 3381-3400.

[20] O. Martin. A modified variational iteration method for the analysis of viscoelastic beams. Applied Mathematical Modelling, 2016, 40(17-18): 7988-7995.

[21] D.D. Demir, N. Bildik, B.G. Sinır. Linear dynamical analysis of fractionally damped beams and rods. Journal of Engineering Mathematics, 2014, 85(1): 131-147.

[22] S. Mareishi, M. Rafiee, X.Q. He, K.M. Liew. Nonlinear free vibration, postbuckling and nonlinear static deflection of piezoelectric fiber-reinforced laminated composite beams. Composite Part B: Engineering, 2014, 59: 123-132.

[23] S. Shiralashetti, R.A. Mundewadi. Modified wavelet full-approximation scheme for the numerical solution of nonlinear volterra integral and integro-differential equations. Applied Mathematics \& Nonlinear Sciences, 2016, 1(2): 529-546.

[24] Y.M. Chen, Y.N. Sun, L.Q. Liu, X.H. Ke. Solving a class of fractional differential equations based on quasi-legendre polynomial. Computational Mathematics, 2015(1): 24-32.

[25] J. Freundlich. Vibrations of a simply supported beam with a fractional viscoelastic material model-supports movement excitation. Shock and Vibration, 2013, 20(6): 383-388. 